\title{
Pulsars in Globular Clusters
}

\author{
Scott M. Ransom
}

NRAO, 520 Edgemont Rd., Charlottesville, VA, 22903 USA, email: sransom@nrao.edu

\begin{abstract}
Globular clusters produce orders of magnitude more millisecond pulsars per unit mass than the Galactic disk. Since the first cluster pulsar was uncovered 20 years ago, at least 138 have been identified - most of which are binary millisecond pulsars. Because their origins involve stellar encounters, many of the systems are exotic objects that would never be observed in the Galactic disk. Examples include pulsar-main sequence binaries, extremely rapid rotators (including the current record holder), and millisecond pulsars in highly eccentric orbits. These systems are allowing new probes of the interstellar medium, the equation of state of material at supra-nuclear density, the masses of neutron stars, and globular cluster dynamics.
\end{abstract}

Keywords. pulsars: general, globular clusters: general

\section{Introduction}

The first globular cluster (GC) pulsar was identified 20 years ago in the cluster M28 after intense efforts by an international team (Lyne et al. 1987). Since then at least 138 GC pulsars $\dagger$, the vast majority of which are millisecond pulsars (MSPs), have been found. Finding these GC pulsars has required high-performance computing, sophisticated algorithms, state-of-the-art instrumentation, and deep observations with some of the largest radio telescopes in the world, primarily Parkes, Arecibo, and the Green Bank Telescope (GBT). The payoff has been an extraordinarily wide variety of science.

Low-Mass X-ray Binaries (LMXBs) have been known to be orders-of-magnitude more numerous per unit mass in GCs as compared to the Galactic disk since the mid-1970s (Katz 1975, Clark 1975). This overabundance is due to the production of compact binary systems containing primordially-produced neutron stars via stellar interactions within the high-density cluster cores. Since LMXBs are the progenitors of MSPs, this dynamicsdriven production mechanism also applies to them, and it has made GCs (particularly the massive, dense, and nearby ones) lucrative targets for deep pulsar searches.

Camilo \& Rasio (2005) produced an excellent review of the first 100 GC pulsars in 2005. This current review provides a significant update to Camilo \& Rasio as it concentrates on the advances made (primarily with the GBT) within the past several years, including almost 40 additional pulsars and over 50 new timing solutions.

\section{Basic Properties of GC Pulsars}

There are currently 138 known pulsars in 25 different GCs. Over 100 GC pulsars have been found in the past 10 years, with almost 60 of these coming in the last 4 years from searches using the GBT (see Figure 1). The three clusters Terzan 5, 47 Tucanae, and M28 account for approximately half of these pulsars, with 33, 23, and 11 pulsars in each cluster respectively. Of the known pulsars, 80 are members of binary systems, 50 are isolated, and 8 are as yet undetermined.

$\dagger$ For an up-to-date catalog of known GC pulsars, see Paulo Freire's website at http://www. naic.edu/ p preire/GCpsr.html 


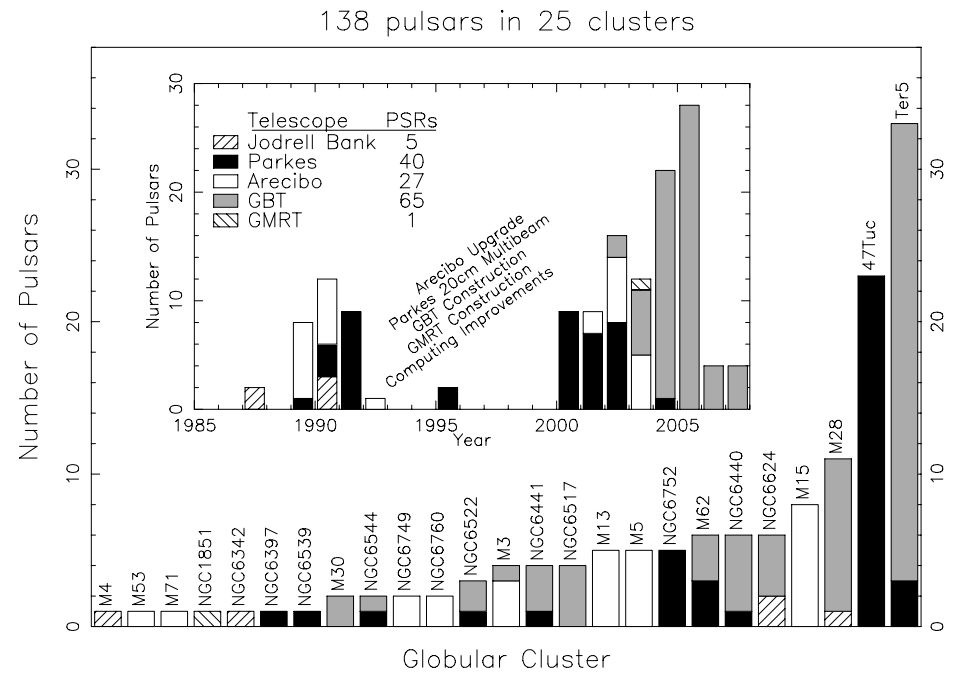

Figure 1. The number of pulsars per globular cluster and (inset) a timeline of their discovery.

Almost $90 \%$ of GC pulsars are true MSPs with spin periods $P_{\text {spin }}<20 \mathrm{~ms}$ (see Figure 2). Their spin properties seem consistent with the standard "recycling" scenario (e.g. Alpar et al.1982), with surface magnetic field strengths of $B \sim 10^{8-9} \mathrm{G}$ and characteristic ages of $\tau_{c} \sim 10^{9-10} \mathrm{yrs}$. Most of the rest of the pulsars are partially recycled. However, there are several seemingly very out-of-place "normal" radio pulsars with $P_{\text {spin }}>0.2 \mathrm{~s}$ and $\tau_{c} \sim 10^{7}$ yrs as well (Lyne et al. 1996).

There are at least four distinct groups of binary GC pulsars (see Figure 2). The first two are similar to the binary MSPs found in the Galactic disk. The "Black Widows" have very low mass companions $\left(M_{c} \lesssim 0.04 \mathrm{M}_{\odot}\right)$ and orbital periods of several hours, while the "normal" low-mass binary MSPs (LMBPs) likely have Helium white dwarf (WD) companions of mass $M_{c} \sim 0.1-0.2 \mathrm{M}_{\odot}$ and orbital periods of several to tens of days. The Black Widow systems are relatively much more common in GCs $(\sim 25 \%$ of the binaries) than in the Galactic disk, though ( $\sim 4 \%$ of the binaries).

The other two groups of binary GC pulsars are possibly unique to clusters and their formation therefore likely depends on the high stellar densities and interactions found in the cores of GCs. Approximately 10\% of GC binaries appear to have "main sequence"like companions which show irregular eclipses, erratic timing, and often have hard Xray and/or optical counterparts. The prototype system is J1740-5340 in NGC 6397 (D'Amico et al. 2001). Finally, $\sim 20 \%$ of the known GC binaries have highly eccentric orbits (with $e>0.1$ ). The standard recycling scenario produces circular orbits due to tidal interactions during mass transfer, and so the large eccentricities are probably either induced during multiple stellar interactions with passing stars (e.g. Rasio \& Heggie 1995) or produced directly during an exchange encounter with another star or binary. Over the past several years the numbers of pulsars in each of these two groups have grown dramatically. This is likely due to the fact that recent surveys have successfully probed many of the most massive and dense clusters in the Galaxy where these systems are preferentially produced (i.e. M28, M62, 47 Tucanae, Terzan 5, NGC 6440, and NGC 6441).

\section{Searching for Cluster Pulsars}

The signal-to-noise ratio from a radio observation towards a (perhaps unknown) pulsar is $\propto S_{\nu} A_{e} T_{s y s}^{-1} \sqrt{B_{\nu} t_{o b s}}$ (e.g. Lorimer \& Kramer 2004). $S_{\nu}$ is the pulsar's flux density at 

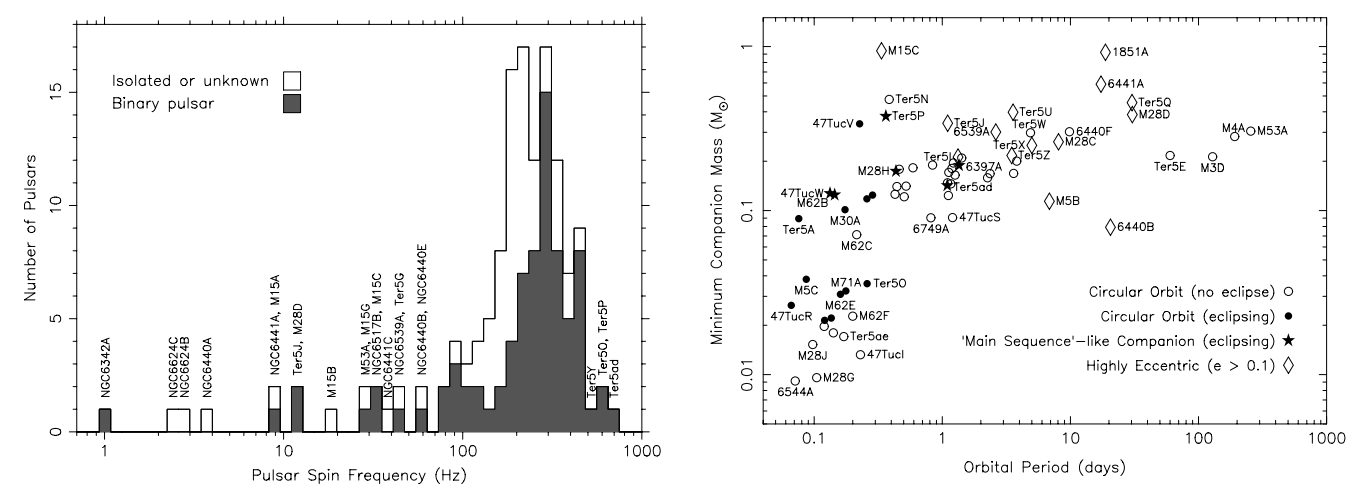

Figure 2. (Left) Spin frequency histogram of the 138 currently known GC pulsars. Eighty of the pulsars are confirmed members of binaries, 50 are isolated, and 8 are as yet undetermined. It is interesting to note that the binary MSPs seem to spin more rapidly on average than the isolated MSPs. Perhaps this is an indication that they are in general younger (i.e. more recently recycled) than the isolated MSPs. Such an explanation makes sense if all isolated MSPs originally come from binaries and therefore must destroy their companions over time. (Right) Orbital period $P_{o r b}$ vs. minimum companion mass $\left(M_{c, m i n}\right.$; assuming a pulsar mass of $\left.1.4 \mathrm{M}_{\odot}\right)$ for the 70 binary GC pulsars with well-determined orbits. All 6 pulsars with "main sequence"-like companions and 14 of the known pulsars in highly eccentric $(e>0.1)$ orbits are labeled, as well as many more "normal" binary systems. Pulsars in "NGC" clusters are labeled without "NGC" to save space. The large grouping of pulsars with $P_{\text {orb }}<1$ day and $M_{c, \min }<0.04 \mathrm{M}_{\odot}$ are the so-called "Black Widow" systems. The lack of pulsars in the lower right portion of the diagram is not due to selection effects, as those pulsars (if they existed) would be relatively easy to identify during searches or through timing observations. M15C is the only confirmed GC double neutron star system (Jacoby et al. 2006) while Ter5N is likely the only known GC pulsar with a Carbon-Oxygen WD companion (Ransom et al. 2005).

the observing frequency $\nu . A_{e}$ is the effective collecting area of the telescope. $T_{s y s}$ is the system temperature, which at the $\sim \mathrm{GHz}$ radio frequencies of interest is roughly the sum of the receiver temperature (typically $15-25 \mathrm{~K}$ ), the $2.7 \mathrm{~K}$ cosmic microwave background, and the Galactic synchrotron background $\left(T_{G a l} \propto \nu^{-2.6}\right.$ and typically a few to tens of $\mathrm{K}$ depending on sky position and observing frequency $\nu$ ). $B_{\nu}$ is the radio bandwidth used for the observation and $t_{o b s}$ is the observation duration. In addition, signal-to-noise ratios improve when a pulsar has short duration pulsations compared to its pulse period (i.e. a small pulse duty cycle). If the pulses are smeared or broadened in time, perhaps by uncorrected orbital motion or interstellar medium effects, signal-to-noise ratios during searches can be reduced to effectively zero.

\subsection{The Problems with Cluster Distances}

MSPs are intrinsically very faint radio sources. Because of this, all of the wide-area Galactic pulsar surveys conducted to date have been severely sensitivity limited for MSPs (many have been instrumentation limited as well). Of the $\sim 60$ known Galactic MSPs, $\sim 80 \%$ are within $2 \mathrm{kpc}$ of the Sun. In contrast, the nearest GCs (M4 and NGC 6397) are just over $2 \mathrm{kpc}$ from the Sun, and most, including the best targets for pulsar searches, are at distances of $5-15 \mathrm{kpc}$ (Harris 1996).

In addition to the inverse square law problem, the large distances to GCs often (especially for the bulge clusters near the Galactic center) imply large column densities of the interstellar medium (ISM). The ionized ISM causes frequency-dependent dispersion of radio waves $\left(\propto \nu^{-2}\right)$, scatter-broadening of the radio pulses $\left(\propto \nu^{-4.4}\right)$, and for certain clusters dramatic fluctuations of observed pulse intensity due to diffractive scintillation (e.g. Camilo et al.2000). These ISM effects, as well as a substantially reduced Galactic 
synchrotron background, have pushed typical observing frequencies from $\sim 400 \mathrm{MHz}$ in the early $1990 \mathrm{~s}$ up to $1.3-2 \mathrm{GHz}$ in the past decade. At these frequencies, especially with much wider observing bandwidths available (hundreds of $\mathrm{MHz}$ ), significant sensitivity gains have been realized despite the usually steep radio spectra of the pulsars themselves (flux densities $S_{\nu} \propto \nu^{\alpha}$ with $-3 \lesssim \alpha \lesssim-1$ and $\langle\alpha>\sim-1.8$; Maron et al.2000).

\subsection{Searching for Binaries}

Since most GC pulsars are in binariest, orbital motion causes Doppler variations of the observed pulsation frequencies during an observation. If uncorrected, these variations can make even very bright MSPs undetectable. However, correcting for unknown orbital motion identically would be extremely computationally expensive and so current searches only account for linear changes in apparent spin frequencies (i.e. constant $\dot{f}$ ). These "acceleration" searches (e.g. Johnston \& Kulkarni 1991) are valid when the orbital period is much longer than the observation duration $\left(P_{\text {orb }} \gtrsim 10 t_{o b s}\right)$.

Acceleration searches add an extra dimension to the traditionally two-dimensional phase space of dispersion measure DM $\ddagger$ and spin frequency $f$ over which one must search for pulsars. Since the number of trials in the acceleration or $\dot{f}$ dimension is proportional to $t_{o b s}^{2}$, the long observations used to improve the sensitivity of GC searches greatly increase their computational costs. Typically, searches for the first pulsar in a cluster are made using a large range of likely DMs but only a limited range of accelerations or $\dot{f}$. Once the first pulsar is found and the rough DM toward the cluster is known, a much smaller range of DMs is searched, but with a much larger range of possible accelerations for additional pulsars.

As an example, to properly search a single $7 \mathrm{hr}$ GBT observation of Terzan 5 (where the $\mathrm{DM}$ is known to $\sim 5 \%$ ) with a full range of acceleration searches, requires approximately one CPU-year of processing on state-of-the-art CPUs. However, it is important to realize that without acceleration searches (or other advanced binary search techniques such as Dynamic Power Spectra; Chandler 2003), the majority of the binary GC MSPs that have been uncovered over the past decade would simply not have been found.

\subsection{A Renaissance in 2000}

The above paragraphs summarize why GC pulsar searches require long integrations (sensitivity) at $\mathrm{GHz}$ frequencies (minimize ISM effects), using the largest telescopes (collecting area), the best receivers (wide bandwidths and low system noise), and large amounts of high-performance computing (acceleration searches). The dramatic increases in the numbers of known GC pulsars beginning in 2000 (see Figure 1) resulted from significant improvements in each of these areas. First, new low-noise and wide-bandwidth $\left(B_{\nu} \sim 300 \mathrm{MHz}\right)$ observing systems centered near $1.4 \mathrm{GHz}$ became available at Parkes and Arecibo. Second, the rise of affordable cluster-computing allowed acceleration searches to be conducted at investigator institutions rather than at special supercomputing sites. The first major success from these improvements (and a significant driver for further efforts) was the discovery of 9 new binary MSPs in 47 Tuc (Camilo et al. 2000).

The third and perhaps most important improvement was the completion of the GBT in 2001. With its state-of-the-art receivers, approximately three times greater $A_{e}$ than Parkes, and the ability to observe over $80 \%$ of the celestial sphere, it is perfectly suited to make deep observations of GCs. By the end of 2003, a fantastic wide-bandwidth $\left(B_{\nu} \sim 600 \mathrm{MHz}\right)$ system centered near $2 \mathrm{GHz}$ became available which provided $5-20$ times

$\dagger$ The current binary fraction of $\sim 60 \%$ is a lower limit since finding isolated pulsars is much easier than finding binaries.

$\ddagger \mathrm{DM}$ is the integrated electron column density along the line-of-sight to a pulsar. 
more sensitivity for MSPs in certain GCs in the Galactic bulge than the $1.4 \mathrm{GHz}$ system used at Parkes. The discovery of 30 new MSPs in Terzan 5 (Ransom et al. 2005), a cluster previously extensively searched at Parkes, including the fastest known MSP (J1748-2446ad aka Ter5ad; Hessels et al.2006), were some of the first results. Pulsar surveys of many additional GCs using the same system are ongoing.

\subsection{Future Cluster Pulsar Surveys}

Recent work on the luminosities $L$ of GC pulsars (Hessels et al. 2007) has confirmed earlier results (e.g. Anderson 1992) suggesting that the luminosity distribution roughly follows a $d \log N=-d \log L$ relation. In addition, this work implies that we currently observe only the most luminous pulsars in each cluster. Together, these facts indicate that our current GC pulsar surveys are completely sensitivity limited such that even marginal improvements in search sensitivities will result in new pulsars $\dagger$. The history of GC pulsar searches has directly demonstrated this fact many times.

However, there seems to be little likelihood of making very large improvements in GC pulsar search sensitivities over the next several years. Most of the variables in the signal-to-noise equation are already nearly optimal (e.g. $T_{s y s}, B_{\nu}, t_{o b s}$, and $\nu$ ). Dramatic improvements in sensitivities and therefore pulsar numbers will almost certainly require a new generation of larger telescopes (i.e. larger $A_{e}$ ) such as FAST or the SKA $\ddagger$.

\section{Which clusters have pulsars?}

Figure 1 shows the number of pulsars in each of the GCs with known pulsars. Currently there are 10 clusters with 5 or more pulsars and 3 clusters with 10 or more pulsars: M28 with 11, 47 Tucanae with 23, and Terzan 5 with 33. Camilo \& Rasio (2005) pointed out that there are very few clear correlations between cluster parameters and the numbers of known pulsars. In fact, the only simple properties that seem to be related to the number of known pulsars are the total mass of the cluster (which likely influenced how many neutron stars were originally retained) and the distance $D$ to the cluster (since all GC pulsar searches are currently sensitivity limited). However, even these indicators have exceptions. For example, $\omega$ Centauri has been searched extensively but unsuccessfully with the Parkes telescope, yet it is one of the nearest and most massive GCs in the Galactic system.

A more sophisticated indicator of which clusters may contain more LMXBs and therefore MSPs is the predicted stellar interaction rate $\Gamma_{c}$ in the cores of the clusters, where LMXBs and MSPs are likely formed. Pooley et al. (2003) showed a strong correlation between the number of X-ray sources in a cluster and its $\Gamma_{c}$, which they expressed as $\Gamma_{c} \propto \rho_{0}^{1.5} r_{c}^{2}$, where $\rho_{0}$ is the central density and $r_{c}$ is the core radius. We can attempt to adjust the indicator to account for our senstivity issues by ranking clusters by $\Gamma_{c} D^{-2}$. Using this metric, we find that many of the clusters with numerous pulsars are near the top of the list, including 47 Tuc, Terzan 5, M62, NGC 6440, NGC 6441, NGC 6544, M28, and M15. Also near the top are several others which likely contain numerous pulsars but whose positions near or behind the Galactic center region (and therefore large amounts of ISM) make searches very difficult (e.g. NGC 6388 and Liller 1; Fruchter \& Goss 2000).

It is important to realize, though, that because of limited amounts of telescope time, pulsar searchers have specifically targeted those clusters near the top of the $\Gamma_{c} D^{-2}$

$\dagger 47$ Tuc is a possible exception to this rule as deep radio imaging (McConnell et al.2004) and X-ray observations (Grindlay et al. 2002) indicate that scintillation may have already allowed the identification of nearly all of the observable MSPs in the cluster.

$\ddagger$ FAST: http://www.bao.ac.cn/LT/, SKA: http://www.skatelescope.org 
list first. Therefore, clusters further down that list may simply not have known pulsars because they haven't been searched to the same sensitivity levels as the clusters near the top of the list. Until we have a large number of GCs, independent of their $\Gamma_{c} D^{-2}$ values, searched to sensitivities comparable to the recent GBT $2 \mathrm{GHz}$ surveys, it will be difficult to determine just how good of a predictor $\Gamma_{c} D^{-2}$ really is.

The recent $1.4 \mathrm{GHz}$ survey of all $22 \mathrm{GCs}$ within $50 \mathrm{kpc}$ and visible with Arecibo (Hessels et al. 2007) is a good example of the type of surveys we need. The Arecibo survey found 11 new MSPs, the majority of which are in clusters with fairly average values of $\Gamma_{c} D^{-2}$. No pulsars were found (or have ever been found) in clusters with very low central luminosity densities, $\rho_{0}<10^{3} \mathrm{~L}_{\odot} \mathrm{pc}^{-3}$. A similar unbiased survey of $\sim 60$ GCs at $1.4 \mathrm{GHz}$ using the Parkes telescope has uncovered 12 new pulsars (D'Amico et al.2001, Possenti et al.2001) in 6 GCs. Unfortunately, the limited sensitivity of that survey does not rule out even relatively bright MSPs in many of the clusters, thereby making it difficult to draw conclusions about cluster properties and their pulsar populations.

\subsection{What pulsars are in those clusters?}

We can compare the pulsar populations in the best studied clusters, such as Terzan 5 and 47 Tucanae, to attempt to determine if the properties of the clusters affect their pulsars. Two of the simplest things to compare are the spin-period distributions and the binary populations, both of which do show significant differences.

The spin-period distribution of the Terzan 5 pulsars is significantly flatter than that of the 47 Tuc pulsars with more faster and more slower pulsars. In fact, Ter 5 contains 5 of the 10 fastest known spinning pulsars (Hessels et al. 2006), while 47 Tuc contains only one in the top ten. Likewise, 47 Tuc has no MSPs slower than $\sim 8 \mathrm{~ms}$, whereas Ter 5 has six. A Kolmogorov-Smirnov test suggests a $<10 \%$ chance that the two period distributions were drawn from the same parent distribution. As for the binary populations, Ter 5 has only two known "Black-Widow" systems compared to five in 47 Tuc. Yet Ter 5 has six highly-eccentric binaries compared to none in 47 Tuc. Are these differences related to the current interaction rates in the cluster cores (Ter 5's is 2-3 times that of 47 Tuc's) or perhaps the "epochs" when the MSP production rates peaked?

The pulsars in M28, NGC 6440, and NGC 6441 are more similar to those in Ter 5 than to those in 47 Tuc, both in terms of their spin periods (i.e. flatter distributions) and their binary parameters (with a broader mix of different systems). It is interesting to note, though, that the 10 known pulsars in the very similar clusters NGC 6440 and NGC 6441 rotate on average significantly slower than those in either Ter 5 or 47 Tuc. Only one MSP is faster than $5 \mathrm{~ms}$, and half are slower than $13 \mathrm{~ms}$ (Freire et al. 2007b).

\section{Timing of Cluster Pulsars}

While it is obviously essential to find the pulsars in GCs to do any science with them, without detailed follow-up observations, and in particular pulsar timing solutions, the amount of science one can do is severely limited. For most GC pulsars the extraordinary precision provided by MSP timing provides $\sim 0.1^{\prime \prime}$ and often significantly better astrometric positions (crucial for multi-wavelength follow-up, e.g. Grindlay et al.2002), extremely precise Keplerian orbital parameters for binaries, and measurements of the pulsar's apparent spin period derivative. For some pulsars, particularly those in eccentric orbits, certain post-Keplerian orbital parameters can be measured (e.g. Lorimer \& Kramer 2004) which determine or constrain the pulsar and/or companion star masses. Establishing timing solutions for as many GC pulsars as possible allows us to use them individually and collectively to probe a wide variety of basic physics and astrophysics. 
Over the past two years, the number of GC pulsars with timing solutions (currently 107) has almost doubled, resulting in many interesting new results.

\subsection{Ensembles of pulsars}

The largest ensemble of pulsars with timing solutions in a GC are the 32 in Terzan 5 (only Ter5U, a weak eccentric binary, remains without a solution). However, there are five other GCs with five or more pulsars with timing solutions (47 Tuc, Freire et al.2003; M28, Bégin 2006; M15, Anderson 1992; NGC 6440, Freire et al.2007b; and NGC 6752, Corongiu et al. 2006). These pulsar ensembles can produce unique science.

Probes of Ionized ISM and Intra-Cluster Medium The precise DMs (errors $<0.1 \mathrm{pc} \mathrm{cm}^{-3}$ ) and timing positions for 32 pulsars in Ter 5 have recently allowed a unique probe of the ionized Galactic ISM between us and the cluster on parsec scales (Ransom 2007). A calculation of the DM structure function indicates that the fluctuations in the ISM on $0.2-2$ pc scales roughly follow those predicted for Kolmogorov turbulence. Earlier work on 47 Tuc using the pulsar positions, accelerations (see Figure 3), and DMs provided the first definitive measurement of ionized gas within a GC (Freire et al. 2001).

Statistical Neutron Star Mass Measurement Using the projected offsets of pulsars from their cluster centers and a model for how relaxed components of different masses should be distributed within a cluster, it is possible to statistically measure the masses of the pulsar systems $M_{p}$ (Heinke et al. 2003). Figure 3 shows the 107 GC pulsars with timing positions split into two different groups: 1) isolated pulsars or binary pulsars with very low-mass companions $\left(M_{c, \text { min }} \lesssim 0.05 \mathrm{M}_{\odot}\right)$ and 2$)$ binary pulsars with more massive companions. Surprisingly, the "isolated" systems seem more centrally condensed than the (supposedly more massive) binary systems. Fits of the observed distributions give $q=M_{p} / M_{\star} \sim 1.5$ for the binaries and $q \sim 1.7$ for the "isolated" systems. Assuming that the dominant stars in the cluster cores have mass $M_{\star}=0.9 \mathrm{M}_{\odot}$ implies binary system masses of $M_{p, b i n} \sim 1.35 \mathrm{M}_{\odot}$ and larger masses $M_{p, \text { iso }} \sim 1.53 \mathrm{M}_{\odot}$ for the "isolated" pulsars. The reason for this difference in mass segregation is currently unknown.

Cluster Proper Motions The very precise positions available from MSP timing allow individual pulsar proper motions given regular observations over 5-10 years. The measurement of several pulsar proper motions from a single cluster allows a measurement of the proper motion of the GC itself. Currently this has been accomplished for three clusters (47 Tuc, Freire et al.2003; M15, Jacoby et al.2006; and NGC 6752, Corongiu et al.2006), and several more will likely be measured within the next couple of years. Such measurements are very important for determining the Galactic orbits of GCs and predicting the effects of tidal stripping and/or destruction. Measuring cluster proper motions is very difficult in the optical (using HST, for instance), especially for the Galactic bulge clusters which are distant and plagued by extinction.

Cluster Dynamics The projected positions of the pulsars with respect to the cluster centers as well as measurements of their period derivatives (which are usually dominated by gravitational accelerations within the GC; see Figure 3) provide a sensitive probe into the dynamics of the cluster and even constrain the mass-to-light ratio near the cluster center (Phinney 1992). These measurements can provide evidence for the presence (or absence) of black holes in the cores of the clusters (D'Amico et al. 2002). Cluster dynamics can also eject pulsars to the outskirts of the clusters or even entirely (Ivanova et al. 2007). A recently uncovered example of such a system is M28F, a bright (for a GC MSP) isolated pulsar located almost $3^{\prime}$ from the center of M28. That offset is larger than for any other GC pulsar except NGC 6752A (Corongiu et al. 2006), where exotic ejection mechanisms have been invoked to explain its position (e.g. Colpi et al. 2003). 

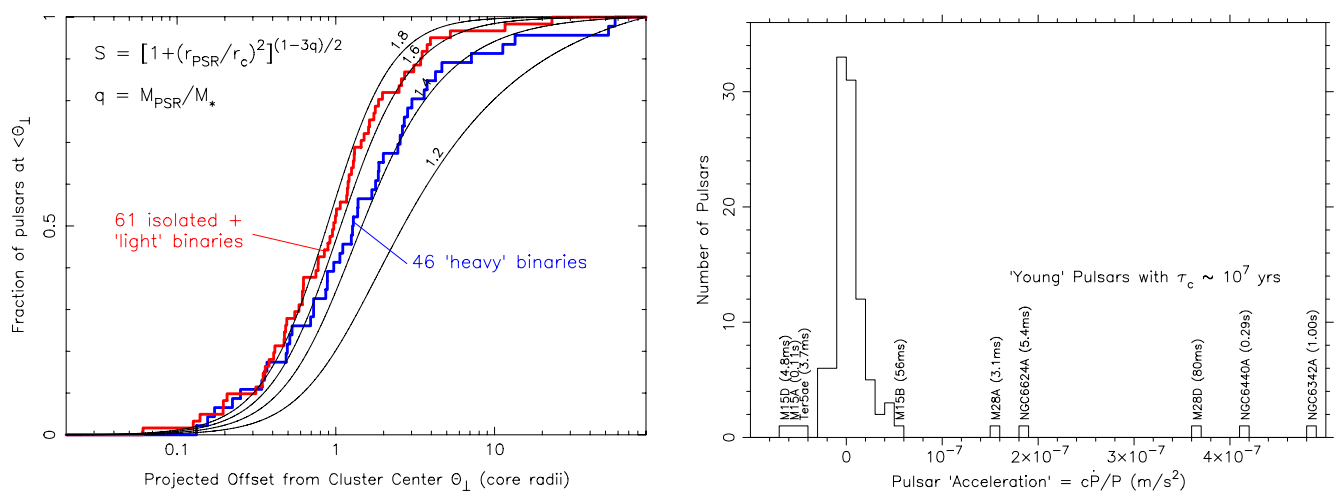

Figure 3. (Left) Radial distribution of "isolated" pulsars (including binaries with very low-mass companions, $\left.M_{c} \lesssim 0.05 \mathrm{M}_{\odot}\right)$ and heavier binary pulsar systems. The numbers on the black lines are $q=M_{p} / M_{\star}$ values where $M_{p}$ is the pulsar system mass and $M_{\star}$ is the dominant stellar component mass in the GC cores $\left(M_{\star} \sim 0.9 \mathrm{M}_{\odot}\right)$. Surprisingly, the "isolated" systems appear more centrally condensed (and therefore possibly more massive) than the binaries. (Right) Histogram of observed GC pulsar "accelerations" $\left(c \dot{P}_{o b s} / P_{o b s}\right)$. The observed acceleration is the sum of the pulsar's intrinsic spin-down (i.e. $c \dot{P}_{\text {int }} / P_{\text {int }}$ ), an apparent acceleration due to proper motion, and gravitational accelerations from both the Galaxy and the GC (Phinney 1992, 1993). The proper motion and Galactic terms are typically small compared to the others. Since GC pulsars are usually recycled and have small intrinsic "accelerations" $\left(\sim 10^{-9} \mathrm{~m} \mathrm{~s}^{-2}\right)$, the GC gravitational term $\left(\sim\left|10^{-8}\right| \mathrm{m} \mathrm{s}^{-2}\right)$ typically dominates. This is apparent in the figure by a nearly symmetric clustering of pulsars around zero acceleration. Pulsars on the observer's side of a GC receive positive accelerations while those on the far side receive negative accelerations and appear to spin more rapidly with time. The five pulsars with observed accelerations $>10^{-7} \mathrm{~m} \mathrm{~s}^{-2}$ are anomalously young (characteristic ages $1 \times 10^{7} \lesssim \tau_{c} \lesssim 3 \times 10^{7}$ years) with intrinsic accelerations much larger than the maximum possible gravitational accelerations from their GCs. Apparently GCs continue to produce such systems (e.g. Ivanova et al.2007). The three pulsars with the most negative accelerations provide unique probes of the central dynamics (and lower limits on the mass-to-light ratios) of their parent GCs (e.g. Anderson 1992).

\subsection{Individual Exotic Pulsars}

Ensemble studies are interesting, but many GC pulsars are worth studying individually.

Young and Slow Pulsars A handful of slow, "normal" ( $\left.\tau_{c} \sim 10^{7} \mathrm{yrs}\right)$ pulsars have been known in GCs for some time: B1718-19 aka NGC 6342A (Lyne et al. 1993), B1820-30B aka NGC 6624B (Biggs et al. 1994), and B1745-20 aka NGC 6440A (Lyne et al. 1996). Recently, at least one more slow pulsar has been uncovered, NGC 6624C with $P_{\text {spin }}=$ 0.405 s (Chandler 2003), as well as M28D, an $80 \mathrm{~ms}$ binary pulsar that is definitely "young" (see Figure 3). The slow pulsars, which have likely not been through any recycling, must have formed relatively recently even though all of the massive stars would have gone supernova $10^{10} \mathrm{yrs}$ ago. One possibility is that the pulsars formed via electroncapture supernovae, perhaps via accretion-induced collapse of a massive WD or mergerinduced collapse of coalescing double WDs (Ivanova et al. 2007).

The fastest MSPs Ter5ad is the fastest MSP known, with a spin period of $P_{\text {spin }}=$ $1.396 \mathrm{~ms}$ (Hessels et al. 2006). Its discovery broke the 23-yr-old "speed" record established by the very first MSP known (Backer et al. 1982) and renewed hope for finding a subMSP (a pulsar with $P_{\text {spin }}<1 \mathrm{~ms}$ ). A sub-MSP would provide the most direct and interesting constraints on the properties of matter at nuclear densities and would be of major significance to physics in general (Lattimer \& Prakash 2007). Ter5 also hosts several very rapid rotators (Ter5O at $1.676 \mathrm{~ms}$ and Ter5P at $1.728 \mathrm{~ms}$; Ransom et al. 2005), and it seems likely that the first sub-MSP (if they exist) might be found in a GC. 
"Main-Sequence"-MSP Systems Several pulsars have been recently discovered (including 47TucW, Edmonds et al.2002, Bogdanov et al.2005; M62B, Possenti et al.2003; Ter5P, Ransom et al.2005; Ter5ad, Hessels et al.2006; and M28H, Bégin 2006) which appear to have bloated "main-sequence"-like companion stars much like the prototype system J1740-5340 in NGC 6397 (D'Amico et al.2001; Ferraro et al.2001). These pulsars are eclipsed for large fractions of their orbits and often show irregular eclipses. Timing positions usually associate them with hard X-ray point sources where the X-rays are likely generated via colliding MSP and companion winds. Several of the pulsars have been identified in the optical where they exhibit variability at the orbital period. In addition, at least some of them exhibit highly erratic orbital variability (resulting in several large amplitude orbital period derivatives) likely due to tidal interactions with the bloated companion stars. These systems could be the result of an exchange encounter between a main sequence star and a "normal" binary MSP system. Alternatively, perhaps the companions have recently recycled the pulsars and we are observing newly born MSPs (D'Amico et al.2001; Ferraro et al.2001). Multi-wavelength studies of these systems are difficult (e.g. Bogdanov et al.2005), but they allow constraints on MSP emission mechanisms, winds, evolutionary histories, and even tidal circularization theory.

Highly Eccentric Binaries At least 15 GC pulsar systems are members of eccentric binaries with $e>0.1$, and most of those contain MSPs. In contrast, only a single eccentric binary MSP is known in the Galactic disk. Ten of these systems have been discovered since 2004: six in Terzan 5 (Ransom et al. 2005), two in M28 (Bégin 2006), and one each in NGC 1851 (Freire et al. 2007a) and NGC 6440 (Freire et al. 2007b). Given the angular reference that ellipses provide, pulsar timing easily measures the precession of the angle of periastron or $\dot{\omega}$. For compact companions, $\dot{\omega}$ is dominated by general relativistic effects, and its measurement provides the total mass of the binary system (Lorimer \& Kramer 2004). Timing observations of four of these systems (Ter5I \& J, Ransom et al.2005; NGC 6440B, Freire et al.2007b; and M5B, Freire et al.in prep.) indicate "massive" neutron stars $\left(>1.7 \mathrm{M}_{\odot}\right)$ which constrain the equation-of-state of matter at nuclear densities (Lattimer \& Prakash 2007). Such constraints are impossible to achieve in nuclear physics laboratories here on Earth. In addition, similar measurements for M28C, a $4.15 \mathrm{~ms}$ pulsar in an 8-day orbit with $e=0.85$, indicate that the pulsar is less massive than $1.37 \mathrm{M}_{\odot}$. This is a fairly low mass for a neutron star which must have accreted a substantial amount of material during recycling and will likely constrain recycling models.

Other Exotica There is already one confirmed GC triple system (PSR B1620-26 in M4) which contains an MSP, a WD, and a planetary-mass component (Sigurdsson et al. 2003). Intriguingly, ongoing observations show very strange and seemingly systematic timing residuals from the "isolated" MSP NGC 6440C (Bégin 2006). One explanation for these residuals is the presence of one or more terrestrial-mass planets. Given the strange variety of systems already known in GCs, it is possible that one of the many currently uncharacterized systems could be another unique object.

\section{Prospects for the Future}

Given the wide variety of often unanticipated science that GC pulsars provide, and the fact that we probably currently observe only 10-20\% of all the GC pulsars, the future of the field seems very bright. Search sensitivity improvements with current telescopes will likely uncover tens of new pulsars and next-generation facilities like FAST and the SKA promise to find hundreds. Among these pulsars may be more spectacular exotica, such as MSP-MSP or MSP-black hole binaries, and doubtless many surprising results. 
Acknowledgments Thanks go to my collaborators on recent GC pulsar searches: Steve Bégin, Ryan Lynch, Jennifer Katz, Lucy Frey, Mike McCarty, Ben Sulman, Fernando Camilo, Vicky Kaspi, and especially Jason Hessels, Ingrid Stairs, and Paulo Freire.

\section{References}

Alpar, M. A., et al. 1982, Nature, 300, 728

Anderson, S. B. 1992, PhD thesis, California Institute of Technology

Backer, D. C., et al. 1982, Nature, 300, 615

Bégin, S. 2006, Master's thesis, University of British Columbia

Biggs, J. D., et al. 1994, MNRAS, 267, 125

Bogdanov, S., Grindlay, J. E., \& van den Berg 2005, ApJ, 630, 1029

Camilo, F., et al. 2000, ApJ, 535, 975

Camilo, F. \& Rasio, F. A. 2005, in ASP Conf. Ser., Vol. 328, Binary Radio Pulsars, ed. F. A. Rasio \& I. H. Stairs, 147

Chandler, A. M. 2003, PhD thesis, California Institute of Technology

Clark, G. W. 1975, ApJ, 199, L143

Colpi, M., Mapelli, M. , \& Possenti, A. 2003, ApJ, 599, 1260

Corongiu, A., et al. 2006, ApJ, 653, 1417

D'Amico, N., et al. 2001, ApJ, 561, L89

D'Amico, N., et al. 2002, ApJ, 570, L89

Edmonds, P. D., et al. 2002, ApJ, 579, 741

Ferraro, F. R., et al. 2001, ApJ, 561, L93

Freire, P. C., et al. 2001, ApJ, 557, L105

Freire, P. C., et al. 2003, MNRAS, 340, 1359

Freire, P. C. C., Ransom, S. M., \& Gupta, Y. 2007a, ApJ, 662, 1177

Freire, P. C. C., et al. 2007b, ApJ submitted

Fruchter, A. S. \& Goss, W. M. 2000, ApJ, 536, 865

Grindlay, J. E., et al. 2002, ApJ, 581, 470

Harris, W. E. 1996, AJ, 112, 1487, (http://www.physics.mcmaster.ca/resources/globular.html)

Heinke, C. O., et al. 2003, ApJ, 598, 501

Hessels, J. W. T., et al. 2006, Science, 311, 1901

Hessels, J. W. T., et al. 2007, ApJ in press, arXiv:0707.1602

Ivanova, N., et al. 2007, MNRAS submitted, arXiv:0706.4096

Jacoby, B. A., et al. 2006, ApJ, 644, L113

Katz, J. I. 1975, Nature, 253, 698

Lattimer, J. M. \& Prakash, M. 2007, Physics Reports, 442, 109

Lorimer, D. R. \& Kramer, M. 2004, Handbook of Pulsar Astronomy (Cambridge Univ. Press)

Lyne, A. G., et al. 1987, Nature, 328, 399

Lyne, A. G., et al. 1993, Nature, 361, 47

Lyne, A. G., Manchester, R. N., \& D'Amico, N. 1996, ApJ, 460, L41

Maron, O., et al. 2000, A\&AS, 147, 195

McConnell, D., et al.2004, MNRAS, 348, 1409

Phinney, E. S. 1992, Phil. Trans. Roy. Soc. A, 341, 39

Phinney, E. S. 1993, in ASP Conf. Ser., Vol. 50, Structure and Dynamics of Globular Clusters, ed. S. G. Djorgovski \& G. Meylan, 141

Pooley, D., et al. 2003, ApJ, 591, L131

Possenti, A., et al. 2001, in Aspen Workshop: Compact Objects in Dense Star Clusters, astro-ph/0108343

Possenti, A., et al. 2003, ApJ, 599, 475

Ransom, S. M., et al. 2005, Science, 307, 892

Ransom, S. M. 2007, in ASP Conf. Ser., Vol. 365, SINS - Small Ionized and Neutral Structures in the Diffuse Interstellar Medium, ed. M. Haverkorn \& W. M. Goss, 265

Sigurdsson, S., et al. 2003, Science, 301, 193 